\title{
Validation of the psychometric properties of the Merck vitality quotient: A short self-questionnaire dedicated to « fatigue » evaluation in healthy subject
}

\author{
Allaert Francois-Andre ${ }^{1,2}$, Courau Stephanie ${ }^{3}$, Forestier Anne ${ }^{3}$ \\ ${ }^{1}$ Chair for Medical Evaluation, Methodology department, ESC, Dijon, France \\ ${ }^{2}$ CEN Nutriment, Dijon, France \\ ${ }^{3}$ Merck Medication Familiale, Medical department, Dijon, France
}

\section{Email address}

allaert@cenbiotech.com (A. Allaert)

\section{To cite this article:}

Allaert Francois-Andre, Courau Stephanie, Forestier Anne. Validation of the Psychometric Properties of the Merck Vitality Quotient: A Short Self-Questionnaire Dedicated to «Fatigue » Evaluation in Healthy Subject. International Journal of Nutrition and Food Sciences.

Vol. 3, No. 6, 2014, pp. 515-518. doi: 10.11648/j.ijnfs.20140306.14

\begin{abstract}
Objective: validation of the psychometric properties of the Merck "vitality quotient" questionnaire (VQ) i.e. the measures of its internal consistency, external validation and sensitivity to change. Methods: Prospective observational survey conducted on patients consulting for fatigue and taking Bion3 ${ }^{\circledR}$ during winter period. The VQ includes 10 questions describing subjects' activities and mood, rated from "1: very often" to "10: never". Its comprehensibility and test-retest reliability has been evaluated in one other study. Each patient was asked to fulfil the VQ and the Pichot scale which is a reference scale for fatigue evaluation at inclusion and every month during three months. Internal consistency of the VQ was measured with Cronbach's alpha coefficient, external validation by its correlation coefficient with Pichot scale and its sensitivity to change by the paired $T$ test of its average variations during the study period. Results: 132 subjects $48.2 \pm 13.5$ years old (63.6\% women) were followed. The Cronbach's alpha coefficient has a very high level of 0.93 and therefore indicates a very good internal consistency. The study of the VQ correlation with the fatigue scale of Pichot at baseline shows a high Pearson coefficient of $r=$ $-0.67(p<0.0001)$ and the study of the correlation of their changes during the three months is of $r=-0.75(p<0.0001)$. The VQ is also sensitive to change. After one month the VQ increases from $47.3 \pm 18.5$ to $60.4 \pm 20.5$ and then to $73.5 \pm 18.0$ after 2 months and to $80.9 \pm 18.1$ after 3 months which is highly significant $(p<0,0001)$ and corresponds to improvements of respectively $+27.7 \%,+55.4 \%$ and $+71.0 \%$ of the initial vitality of the subjects taking Bion3 ${ }^{\circledR}$. Conclusion: This study validates psychometrics properties (reliability, external validity and sensitivity to change) of the Merck «vitality quotient» which appears therefore as an effective tool to evaluate food supplements effects in subjects complaining of fatigue, tiredness, exhaustion and expressing a loss of vitality.
\end{abstract}

Keywords: Fatigue, Self-Questionnaire, Validation

\section{Introduction}

Fatigue is one of the most common symptoms for which people are regularly complaining (1) and requiring advice from their pharmacists and/or their medical practitioners (2). This symptom is very subjective and greatly varies between individuals. Several evaluation questionnaires like Pichot (3), or Fatigue Severity (4) or Fatigue assessment scales (5) were developed to quantify it more objectively and to follow its evolution under treatment. These questionnaires have demonstrated their relevance from a health professional perspective: to release the patients' complaints. However, this does not strictly meet the real expectation of healthy subjects who are more expecting to improve their well being and to restore their vitality. The objective of the "vitality quotient" developed by Merck Medication Familiale in France was to measure the physiological and psychological improvement of the loss of vitality perceived by a healthy subject i.e. for whom no organic or psychic disease appears directly at the origin of the fatigue. This work presents the results of a study 
conducted to validate the psychometric properties of the Vitality Quotient (VQ).

\section{Methods}

\subsection{Building of the Questionnaire}

The VQ was created as usual through medical experts' discussion and then with interviews conducted with healthy subjects complaining about fatigue. The objective was to set up a short self questionnaire focused not on symptoms but on what subjects were describing pragmatically as the consequences of their tiredness in their daily life and expressing their loss of vitality. This work leads to a selfquestionnaire of 10 questions. All questions and answers were constructed with high score corresponding to a high level of vitality. Accordingly, answers were describing a possible pragmatic negative effect of the loss of vitality and were scored on a numeric scale from 1 for «Very often» to 10 " Never ". Due to the small number of questions no preliminary study for item reduction was required before psychometric validation study. Analysis with Cronbach's alpha coefficient was sufficient to detect lake of homogeneity or redundant items. The vitality questionnaire is presented in table 1 .

Table 1. Stability of the Vitality Quotient: intraclass correlation coefficient and IC $95 \%$

\begin{tabular}{ll}
\hline & ICC $($ IC 95\%) \\
\hline In the morning, you feel tired even before you start & $0.69[0.45 ; 0.84]$ \\
your day & $0.70[0.47 ; 0.84]$ \\
During the day, you feel the need to take a nap & $0.77[0.58 ; 0.88]$ \\
By late afternoon, before dinner, you feel tired & $0.69[0.45 ; 0.84]$ \\
You're struggling to make your day's activities & $0.84[0.70 ; 0.92]$ \\
Difficulty concentrating & $0.68[0.29 ; 0.77]$ \\
You feel awkward in your actions & $0.92[0.84 ; 0.96]$ \\
You feel the need to limit your leisure activities \\
(sports, music, arts, ..) & $0.87[0.75 ; 0.93]$ \\
You feel the need to be alone for more peace & $0.91[0.82 ; 0.95]$ \\
You feel irritable & $0.84[0.70 ; 0.92]$ \\
You feel less resistant to external aggressions (cold, \\
pollution, ...) & $0.90[0.80 ; 0.95]$ \\
Total score & \\
\hline
\end{tabular}

\subsection{Psychometric Validation of the Questionnaire}

The validation of the psychometric properties of the vitality quotient was conducted through according reference methods $(6,7)$. It includes two steps, each of them corresponding to a specific study.

The first study covered readability and understanding of the questionnaire and its stability/reliability through testretest method. Test-retest reliability was estimated by administering the vitality quotient to the same group of 30 healthy volunteers at two different times. The correlation between the two scores, and between individual questions, indicates the stability of the instrumental measure. Time interval between the original test and the retest was 48 hours exactly, considering that the vitality may quickly change during the day. Readability was assessed by the Flesch test and subjects interviews. Reliability was measured using Intra class coefficient for each question of the vitality quotient and for the global vitality quotient score.

The second step aimed to evaluate the internal consistency, the external validity and the sensitivity to change (responsiveness) of the vitality quotient. Therefore a multicenter observational study using Bion3 ${ }^{\circledR}$ (Food supplement) product was conducted in patients consulting their medical practitioners (GP) during autumn-winter season $2012 / 2013$. In order to represent a real life situation, the population tested was subjects, 18 to 70 years old complaining of winter fatigue and for whom general practitioners considered as appropriate to recommend taking Bion3 ${ }^{\circledR}$ adult tablets. To avoid any modification of the observational condition, no exclusion criterion was applied (except not to meet the inclusion).

Each GP was asked to include the first 5 consecutive subjects meeting these criteria and willing to participate to a three months follow up period using self-questionnaire, fulfilled at inclusion and at the $1^{\text {st }}, 2^{\text {nd }}$ and $3^{\text {rd }}$ month of follow-up.

This self-questionnaire included:

- the Pichot scale for fatigue evaluation (3). This self questionnaire has 8 questions rated 0 for "not at all", 1 for "a little", 2 for "moderately", 3 for "much" and 4 for "extremely" and varies between 0 for « No fatigue » and 32 for « Extreme fatigue »;

- the VQ;

- a Lickert scale dedicated to the evaluation of the patient's satisfaction.

The self questionnaire was strictly anonymous and directly sent to the data center CEN Nutriment with a prepaid envelope at each period of the follow-up.

The internal consistency reliability of the vitality quotient was evaluated using the Cronbach's alpha coefficient which indicates how well the items on a tool fit together. A value higher more than 0.7 is considered as a satisfying result (6). The evaluation of the sensitivity to change (a) and external validity (b) was based on the hypothesis that under effect of Bion $3 \AA$, the vitality quotient would a) increase significantly and b) its increase would be significantly correlated to the significant decrease of the Pichot's scale. Changes in the Pichot's scale and sensitivity to change of the VQ were both evaluated by a paired $\mathrm{T}$ test on repeated measure conducted after 3 months of follow-up. The external validity of the VQ was studied by analyzing its correlation with the fatigue scale of Pichot at baseline, and by measuring the correlation of its change during the three months with the change of the Pichot's scale, using the Pearson coefficient. Data were recorded on capture system and statistical analysis used SAS Version 9.3. The sample size calculation was based on an expected average decrease of at least 2 points of the Pichot's scale, with a standard deviation of 8 , an alpha risk of 0.05 , and a power of $90 \%$. According nQuery Advisor software, the required number of subjects using paired t-test was 171 . 


\section{Results}

\subsection{Understanding and Test-Retest Reliability (Stability)}

30 healthy subjects, $35 \pm 4$ years old, among whom $50 \%$ were women, took part to the study. Readability was assessed by the Flesh Kincaid Grade Level which indicate that the text of the VQ is understandable by people having had at least 8.2 years of education and therefore may be used in the global population without limitations.

Stability, assessed by the intraclass correlation coefficient, showed a very good agreement with a global ICC of 0.90 . The intraclass correlation coefficients for each question are described in table 1 and vary between 0.92 and 0.68 .

\subsection{Internal Consistency, Sensitivity to Change and External Validity}

132 subjects consulting their practitioner for fatigue were followed with self-questionnaire during the three months of the study among the 202 who were included in the study (65.3\%). All were prescribed Bion3 ${ }^{\circledR}$ Adults by their medical practitioners. The characteristics of the subjects followed during the 3 months of the study were not statistically different from those who stopped the study prematurely.

\subsubsection{Subjects' Fatigue and Vitality}

The 132 subjects consulting their practitioner for fatigue were $48.2 \pm 13.5$ years old and $63.6 \%$ of them were women. At inclusion, subjects had a fatigue level estimated to $15.5 \pm 7.2$ on the Pichot Fatigue Scale on a maximum of 32 and a VQ of $47.3 \pm 18.5$ on a maximum of 100 . The details of the answers given to the Vitality Quotient at inclusion are given in table 2 .

Table 2. Description of the Vitality Quotient at inclusion.

\begin{tabular}{llll}
\hline & Mean & SD & Median \\
\hline $\begin{array}{l}\text { In the morning, you feel tired even before you } \\
\text { start your day }\end{array}$ & 4.4 & 2.3 & 4.0 \\
$\begin{array}{l}\text { During the day, you feel the need to take a } \\
\text { nap }\end{array}$ & 4.5 & 2.5 & 4.0 \\
$\begin{array}{l}\text { By late afternoon, before dinner, you feel } \\
\text { tired }\end{array}$ & 4.2 & 2.3 & 4.0 \\
You're struggling to make your day's activities & 4.9 & 2.4 & 5.0 \\
$\begin{array}{l}\text { Difficulty concentrating } \\
\text { You feel awkward in your actions }\end{array}$ & 5.0 & 2.4 & 5.0 \\
You feel the need to limit your leisure & 5.0 & 2.6 & 5.0 \\
activities (sports, music, arts, ...) & 5.5 & 5.0 \\
$\begin{array}{l}\text { You feel the need to be alone for more peace } \\
\text { You feel irritable }\end{array}$ & 5.1 & 2.5 & 5.0 \\
$\begin{array}{l}\text { You feel less resistant to external aggressions } \\
\text { (cold, pollution, ...) }\end{array}$ & 3.7 & 2.6 & 5.0 \\
Total score & 47.3 & 18.5 & 5.0 \\
\hline
\end{tabular}

\subsubsection{Internal Consistency}

The internal consistency of the VQ was measured by the Cronbach alpha coefficient which had a very high level of 0.93 and therefore indicates a very good reliability.

\subsubsection{Sensitivity to Change}

After one month the fatigue level measured with the Pichot's scale decreased from $15.5 \pm 7.2$ to $10.4 \pm 7.0$, and then to $6.0 \pm 5.8$ after 2 months and $3.7 \pm 4.3$ after 3 months $(p<0,0001)$ which corresponds to reductions of respectively $32.9 \%,-61.3 \%$ and $-76.1 \%$ of the initial fatigue level. This result confirms the expected positive effect of Bion $3 \AA$ on fatigue (Figure 1).

The sensitivity to change of the VQ was also described by its evolution at the $1^{\text {st }}, 2^{\text {nd }}$ and $3^{\text {rd }}$ month. After one month, the VQ increased from $47.3 \pm 18.5$ to $60.4 \pm 20.5$ and then to $73.5 \pm 18.0$ after 2 months and to $80.9 \pm 18.1$ after 3 months ( $\mathrm{p}<0,0001)$ which corresponds to improvements of respectively $+27.7 \%,+55.4 \%$ and $+71.0 \%$ of the initial vitality and confirms its sensitivity to change (Figure 2). $60.3 \%$ of the subjects felt an improvement of their vitality of more than $50 \%$ at the third month.

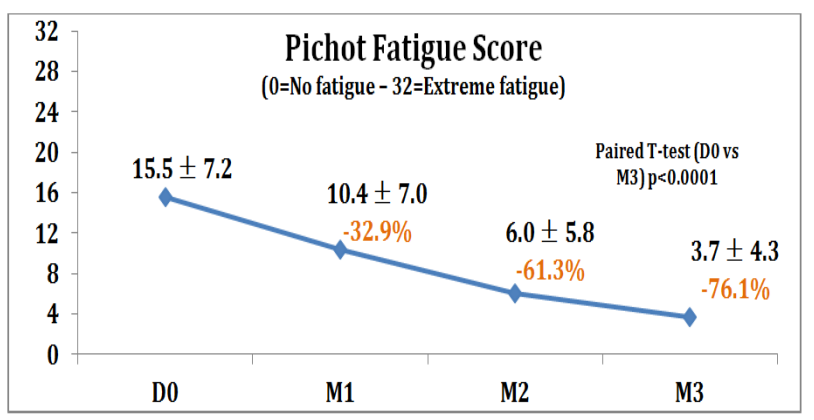

Figure 1. Pichot Fatigue Score evolution: $0=$ No fatigue - 32=Extreme fatigue

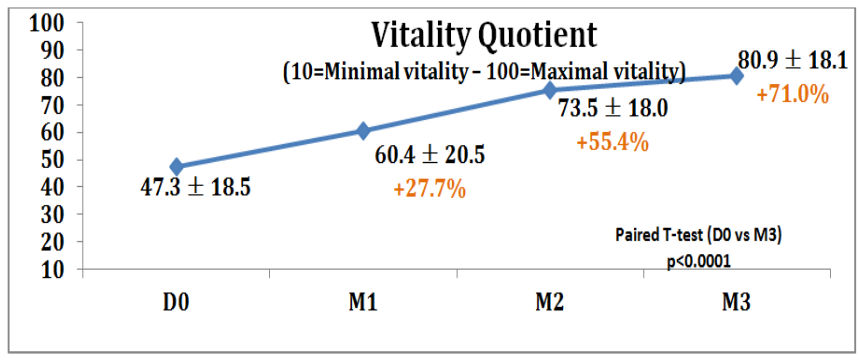

Figure 2. Vitality Quotient evolution

\subsubsection{External Validity}

The external validity of the VQ was studied by analyzing its correlation with the fatigue scale of Pichot at baseline which shows a Pearson coefficient of $r=-0.668(p<0.0001)$ and by analyzing the correlation of its change during the three months and the change of the scale of Pichot which shows a Pearson coefficient of $r=-0.752(p<0.0001)$.

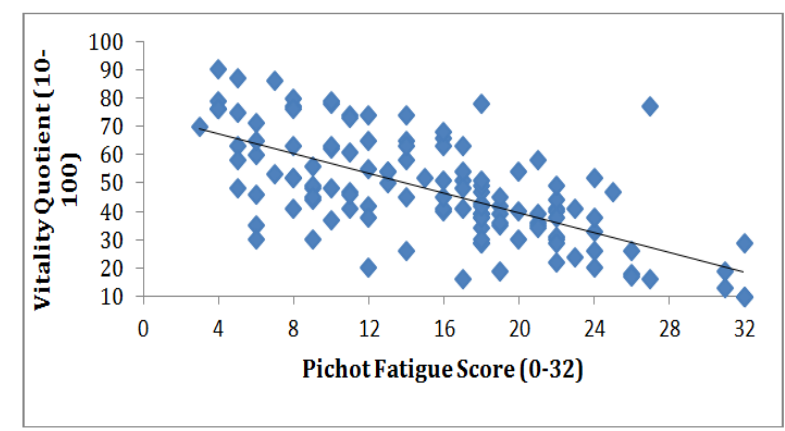

Figure 3. Correlation between VQ and Pichot Fatigue Scale at inclusion 
These results are illustrated in figure 3 which shows an inverse and linear relation between Pichot fatigue scale at inclusion and in figure 4 which shows an increase of the vitality quotient when the fatigue decrease.

\section{Discussion}

This study presents some limitations, the major one lying to drop-out subjects in the observational study. This limitation could impair the effect of the Bion $3 \AA$ impact but a spontaneous answer of two third of subjects during three months is not a bad rate in observational study. Secondly the comparison which shows no difference at inclusion between

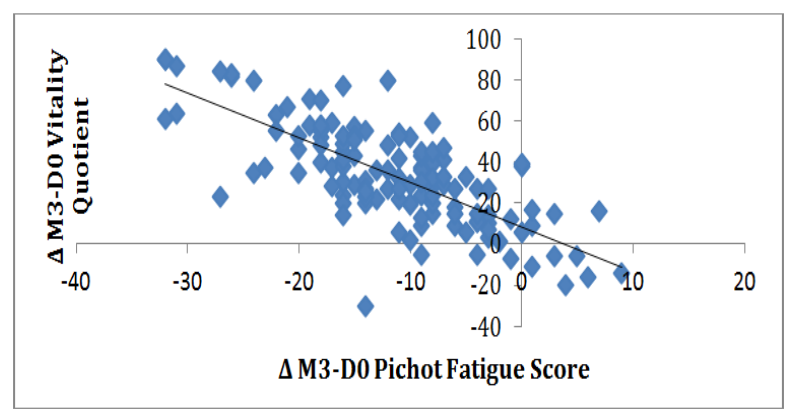

Figure 4. Correlation between Vitality Quotient evolution and Pichot Fatigue Score evolution.

patients followed or not, contributes to eliminate a selection bias. Finally, this may have very small impact on the purpose of that study whose objective was to validate the psychometric properties of the VQ. Indeed, the main objective was to observe a change in the Pichot scale to validate the sensitivity to change of the VQ and to demonstrate its external validity through a correlation with the Pichot's scale. One other limitation was that we did not attempt to make a reduction of the number of questions before testing the VQ but the high result obtain with the Cronbach's alpha coefficient confirmed that it was not necessary. However, the stability of the readability, the testretest were clearly demonstrated in the first study and the second one confirms its internal validity, its sensitivity to change (responsiveness) and it external validity. In particular, it is important to point out that results of the ICC and the Cronbach's alpha coefficient are particularly positives and that the sensitivity to change is very good showing strong variation under Bion3 ${ }^{\circledR}$ intake. The Pearson correlation with the Pichot scale which is around 0.7 may appears more debatable but it comes also from the fact that we want to measure things which are similar but not the same. As explained in the introduction, we want to have a questionnaire more related to healthy subjects than patients and this correlation coefficient seems strong enough to demonstrate external validity but not to high which could indicate that both score don't measure the same thing.

The main advantages of this scale are

- its small number of questions which allows an easy and quick use in the daily practice contrary to some others including a large number of questions

- and above all, its validation in healthy patients whose fatigue did not result from severe pathology as cancer or multiple scleroris $(8,9)$.

\section{Conclusion}

The psychometric properties (reliability, internal consistency, external validity and sensitivity to change) of the Merck Medication Familiale vitality quotient are demonstrated in this study and this evaluation questionnaire appears adapted to evaluate the vitality of healthy subjects either in a daily follow up clinical study either in a self-use perspective.

\section{References}

[1] Pawlikowska T, Chalder T, Hirsch SR, Wallace P, MWright D J, Wessely S C. Population based study offatigue and psychological distress BMJ 1994; 308 : 763-766.

[2] Ridsdale L, Evans A, Jerrett W, Mandalia S, Osler K, Vora H. Patients with fatigue in general practice: a prospective study. BMJ 1993;307: 103-6

[3] Gardenas J. Echelles et outils d'évaluation en médecine générale Échelle de fatigue de Pichot pour l'évaluation de la Fatigue. Le Généraliste- Supplément du N²187; Mars 2002.

[4] Krupp LB, LaRocca NG, Muir-Nash J, Steinberg AD. The Fatigue Severity Scale:application to patients with multiple sclerosis and systemic lupus erythematosis. Arch Neurol 1989;46: 1121-3

[5] De Vries J., Michielsen H., Van Heck GL., Drent M. Measuring fatigue in sarcoidosis: The Fatigue Assessment Scale (FAS). British Journal of Health Psychology (2004), 9, 279-291

[6] DeVon H.A, Block M.E, Moyle-Wright $P$ et al. A Psychometric Toolbox for Testing Validity and Reliability. Journal of Nursing Scholarship 2007 155-164

[7] Pakhale S., Wood-Dauphinee S., Spahija A. Et al. Validation of a new questionnaire with generic and disease-specific qualities: The McGill COPD Quality of Life Questionnaire Can Respir J Vol 2012, 19, 6; 367-372.

[8] Debouverie M, Pittion-Vouyovitch S, Louis S, Guillemin F.Validity of a French version of the fatigue impact scale in multiple sclerosis Mult Scler. 2007 Sep;13(8):1026-32.

[9] Gledhill JA, Rodary C, Mahé C, Laizet C.French validation of the revised Piper Fatigue Scale. Rech Soins Infirm. 2002 Mar;(68):50-65. 